\title{
DETERMINANTS OF HUSBAND'S VIOLENCE AGAINST HIS WIFE IN SOME RURAL AREAS OF SHARKIA GOVERNORATE IN EGYPT
}

\author{
Ayman Ahmed Ecresh \\ Rural Sociology, Dept. of Ag. Economics, Faculty of \\ Agriculture, Zagazig University, Egypt
}

\begin{abstract}
Variables affecting husband's violence against his wife (HVW) in three Egyptian villages in Sharkia governorate having different level of development, are analyzed in this study. Results showed a high prevalence of HVW in the selected rural areas. $71.9 \%$ of wives reported different types of HVW. The main reasons for HVW were: the desire to visit her parents, husband influenced by friends. The main consequences of HVW on wives were related to health effects, both psychological and physical. Multinomial logistic regression results showed that nine out of fifteen variables significant determinants of HVW in this study. These variables together explained $40.1 \%$ of HVW. Four variables were negatively associated with HVW : husband's age at marriage, husband's income, length of cohabitation, and developmental level of village. Five variables were positively associated with HVW: wife's education attainment, husband's education attainment, wife's income, degree of family income adequate for living, and social level of the neighbors.
\end{abstract}

Key words: Husband's violence against his wife, rural women, Egypt.

\section{Introduction}

Violence against women (VAW) is now recognized as a worldwide social problem because it impacts women irrespective of religion or class (Carrillo, 2002, p.S15).Intimate partner violence (IPV) is the most common aspect of VAW (Heise et al., 2002, p.S6). Victims of violence experienced in silence, with some public recognition of their suffering. Recently, women's groups has been organized to claim attention to the forms of physical and psychological abuse of women (Ellsberg and Heise, 2005, p.5). Gradually, VAW has become a substantial human rights issue (Rosche, 2014, p.2).

Researchers have confirmed that IPV is widespread in Egypt Amnesty International (2008), reported that, about 250 women in Egypt have been killed in the first half of 2007 by their husbands or other family members. In the 2014 survey, three in 10 ever-married women have reported some kinds of spousal violence, $25 \%$ physical, $19 \%$ emotional, and 4\%sexual violence (Ministry of Health and Population, El-Zanaty and Associates and ICF International, 2015, 
p.229). Despite the jeopardy of IPV problem, yet few studies have conducted in Egypt in this issue (Diop-Sidibe et al., 2006). Therefore, the current study aims to:

(1) Identifying the characteristics of victims and perpetrators of husband's violence against his wife (HVW) during the last 12 months in the rural of Sharkia governorate in Egypt

(2) Estimating the prevalence of psychological, physical and sexual HVW committed against wives.

(3) Recognizing the reasons of HVW.

(4) Recognizing the consequences of HVW on wives.

(5) Determining the determinants of HVW

\section{Theoretical framework}

The ecological model is the most important approach that attempt to explain and understand the HVW. The model illustrates the influences of multiple four factors of the individual, relationship, community and societal levels in in a particular society. Where no single factor produces partner violence (Dahlberg and Krug, et al., 2002,p. 12; Heise et al., 2002 p.S8 ; Heise,2011, p.vii) (see Fig. 1):

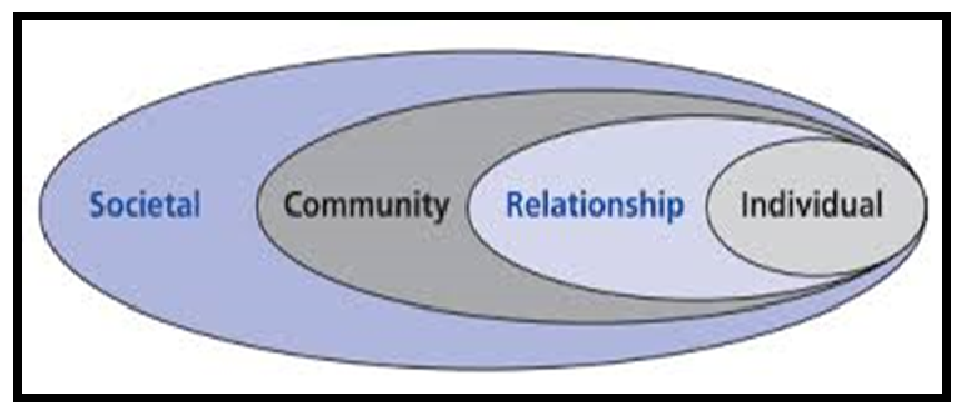

Fig. 1. Ecological model for understanding violence.

Source: Heise et al., 1999, p.8; Dahlberg and Krug, 2002, p.12; CDC, 2004,p.4.

The first circle includes biological and personal history factors that raises the likelihood of individual being a victim or a perpetrator; such as the demographic characteristics (Krug et al., 2002, p.1085).

The second circle represents the relationships factors which abuse take place, such as what occur among family members, other intimate or acquaintance relationship (Heise et al., 1999,p.8).

The third circle, the community contexts, includes the institutions and social structure factors such as social networks and neighborhoods (Heise, 1998, p264). 
The fourth circle represents the larger societal factors, such as economic and social policies, cultural belief systems, and societal norms, that affect HVW levels (WHO, 2010, p19).

\section{Hypotheses}

The reviewed empirical studies led to the following hypotheses: the HVW would decrease with an increase in (1) woman's age; (2) husband's age; (3) wife's age at marriage; (4) husband's age at marriage; (5) wife's education; (6) husband's education; (7) wife's income; (8) husband's income; (9) family income; (10) quality of dwelling; (11) degree of family income adequate for living; (12) length of cohabitation; (13) number of living children; (14) social level of neighbors; (15) developmental level of village.

\section{Methods}

\subsection{Data and Sample}

The present study focused on currently wives who completed at least 12 months of marriage. It was based on a field sample survey. This was conducted in three villages varying in developmental level in Sharkia governorate of Egypt. Sharkia is the third largest governorate, with a population of 5,586,406 inhabitants, and as of 2014 had 497 villages. The three villages were selected by simple multi-stage cluster sampling technique. An index of availability of developmental organizations was established in the first stage, it consists of fourteen items (Bureau of Sharkia governorate, 2014) namely: number of: local units, social units, schools, nurseries, healthy units, youth centers, mosques, churches, veterinary units, police stations, agricultural cooperatives, village banks, post offices, and community development associations. The raw data of this index was transformed to z-scores then to t-scores. The districts of Sharkia governorate were divided by this index to three categories (backward, transitional, and advanced) one district was selected randomly. From these three districts, three villages were also selected randomly by the same technique in the second stage. The backward village was EL-awasga from Hehya district, the transition village was Amriet from Abu Hammad and the advanced village was Mit Suhayl from Menya El-Qamh district.

By Cochran formula (1977) a sample of 360 households were randomly picked from three villages totaling 5737 households. This study identified the sampling frame as all the houses in the selected villages. Households were chosen from the population through a systematic random sampling technique equally allocated. The sampling interval was 16 household. The first households were chosen by simple random sampling. 
The survey questionnaires were administered face-to-face to married women through interviews, during the period NovemberDecember 2014. All questionnaires were completed.

\subsection{Measures}

Similar to the ecological model, the data collection tool of the present study includes social, demographic, economic and cultural variables. The independents variables fall into the first part of the questionnaire in addition to; wife's age, husband's age, wife's age at marriage, husband's age at marriage, wife's education, husband's education, wife's income per Egyptian pond/month (EPM), and husband's income EPM. The second part of the questionnaire contained family income EPM, quality of dwelling, degree of family income adequate for living, length of cohabitation, and number of living children. The third part included social level of neighbors and the fourth part contained the developmental level of village.

Wife's age, husband's age, wife's age at marriage, and husband's age at marriage were measured by years. Wife's education, husband's education were measured by the level of education attained. Wife's income per Egyptian pond/month (EPM), and husband's income EPM were calculated by the average personal monthly income.

Family income EPM was calculated by the total average of monthly income gained by all members of family (wife + husband + children). Quality of dwelling is a relative index. It was calculated based on interviewer-observed assets (type of dwelling ownership, type of building material, type of dwelling's ground, type of cooking's means, presence of sanitation, type of dwelling's coating, degree of modernity of dwelling's furniture, availability of independent kitchen, type of bathroom). To create the quality of dwelling index, each asset was assigned a weight (factor score), and the resulting asset scores were transformed to z-scores then to t-scores. Dwellings were ranked based on these items score and ranked to 3 categories ( $1=\mathrm{bad}, 2=$ middle, $3=$ good). Degree of family income adequate for living was measured by asking whether the total household monthly income was sufficient to met family needs for living or no ( $1=$ not enough, $2=$ enough, $3=$ very enough). Length of cohabitation was measured by the years of cohabitation of current husband and wife. Numbers of living children was calculated by the number of children were living and still single in the family.

Social level of neighbors was measured by asking about the standard of living of people adjacent to the house of respondent ( $1=$ low level, $2=$ middle level, $3=$ high level). Developmental level of village was calculated by the total score of index of availability of developmental 
organizations mentioned above, then ranked to three categories ( $1=$ backward, $2=$ transitional, $3=$ =advanced). All independents variables were categorized (Table 2) after exploratory analysis of the bivariate and multivariate distributions, preserving enough cases in each cell.

The dependent variable of this study; the prevalence of HVW was dichotomous variable. It was defined as $0=$ no form of HVW during the preceding 12 months and $1=$ one or more forms of HVW.

The questionnaire included 3 types of HVW related questions. Each married woman was asked '(Does/did) your (last) husband ever do any of the following things to you: (1) prevent you from talking; (2) belittle or humiliate you; (3) label you with demeaning terms (e.g., "crazy", "animal"); (4) ignore your feelings; (5) blame you for all faults; (6) give you angry stares or looks; (7) threaten you by beating something; (8) prevent you from education; (9) threaten to harm you; (10) humiliate or insult you; (11) prevent you from going out with friends or relatives; (12) threaten to divorce you; (13) criticize your body; (14) prevent you from sleeping; (15) prevent you from food; (16) yelling; (17) prevent you from health care; (18) force you to flee from home now and then; (19) Slap or twist your arm; (20) push you; (21) punch you; (22) kick or drag you; (23) Pull your hair; (24) hit you with harmful object; (25) attempt to choke or strangle you; (26) make bruises in your body; (27) burn you with cigarette; (28) attempt to burn you with fire; (29) use a knife or other weapon to threaten you; (30) break your bones; (31) make internal injuries in your body. (32) forced you to have sex in an unwanted time; (33) label you with sexual labels such as "whore" and "frigid"; (34) force you to unwanted or forced touching; (35) force you to have humiliating sex; (36) forced you to have uncomfortable sex'?. A positive answer of these questions (from 1 to 18) indicated psychological HVW perpetration, positive answer to question (from 19 to 31) indicated physical HVW perpetration and positive answer to question (from 32 to 36 ) indicated sexual HVW perpetration.

After each positive response, currently married women were asked about the frequency of the HVW acts in the 12 months preceding the survey, the reasons for HVW, and the consequences of HVW. To estimate the frequency of HVW acts, total weighted score of each act was calculated by sum scores of frequency of act (never $=0$, few times a year $=1$, few times a week $=2$, few times a month $=3$, every day $=4$ ) for all respondents. These acts were fall in three group of HVW, psychological, physical, and sexual. The weighted average of each group was calculated by divided the total scores of acts in the group on the numbers of acts in the group. 
To recognize the frequency of reasons for HVW, total weighted score of each reason calculated by sum scores of frequency of reason $(0=$ none $1=$ rarely, $2=$ sometimes $3=$ frequently $)$ for all respondents.

To recognizing the frequency of consequences of HVW, total weighted score of each consequence was calculated by sum scores of frequency of consequence $(0=$ never happened, $1=$ rarely, $2=$ sometimes $3=$ often, 4 = always) for all respondents.

\subsection{Statistical analysis}

Past-year prevalence estimates of psychological HVW only, physical HVW only, sexual HVW only, both psychological and physical HVW, both psychological and sexual HVW, both physical and sexual HVW, all forms, any form of HVW were calculated for respondents. Multinomial logistic regression analysis was used to assess which combination of social, demographic, economic, and cultural variables are most associated with HVW and explore to what extent of these variables were independent predictors of total HVW among currently married women. The odds ratio (OR) were derived with 95\% confidence intervals $(\mathrm{Cl})$ using the ratio of abuse prevalence in one category of a variable compared with the ratio in a reference category (first category). Significance of all analyses was set at $\mathrm{P}<0.05, \mathrm{P}<0.01$ or $\mathrm{P}<0.001$. All statistical analyses were conducted using SPSS 16.0 for windows.

\subsection{Reliability Test}

Cronbach's alpha test showed (Table 1) that the internal consistency levels of measures ranged from 0.678 to 0.774 , meaning that all these measures were considered reliable (alpha value greater than 0.60). Some items were removed in these scales in order to improve reliability scores.

Table1. Research Instruments and Reliabilities Obtained

\begin{tabular}{|lcc|}
\hline \multicolumn{1}{|c|}{ Scale Name } & No. of items & Alpha coefficients \\
\hline Psychological HVW & 18 & 0.763 \\
Physical HVW & 12 & 0.774 \\
Sexual HVW & 5 & 0.678 \\
Total HVW & 35 & 0.720 \\
\hline
\end{tabular}

\subsection{Characteristics of sample}

Characteristics of sample in all the three studied villages are presented in Table 2. The largest percentage of total respondents were aged 26-33 years old, married at 19-24 years old, with 6 years or less Length of cohabitation. received secondary education, having 3 children or more, having no personal income, having low level of family income, having middle quality of dwelling, having adequate enough of family income for living and living in middle social level of neighborhood. The largest percentage of their husbands were aged 25-31 years old, married at 21-26 years old, received secondary education and got 100-500. 


\section{Table 2. Percentage Distribution of Characteristics of respondents and their families}

\begin{tabular}{|c|c|c|c|c|c|c|}
\hline \multirow{3}{*}{ Characteristics } & \multicolumn{3}{|c|}{ Developmental level of Village } & \multicolumn{3}{|c|}{ Total HVW (Any form) } \\
\hline & $\begin{array}{c}\text { Backward } \\
\text { village } \\
(n=120)\end{array}$ & $\begin{array}{l}\text { Transitional } \\
\text { village } \\
(n=120)\end{array}$ & $\begin{array}{l}\text { Advanced } \\
\text { village } \\
(n=120)\end{array}$ & $\begin{array}{c}\text { wives } \\
\text { without } \\
\text { lifetime } \\
\text { experiences } \\
\text { of HVW } \\
(n=101)\end{array}$ & $\begin{array}{l}\text { wives with } \\
\text { lifetime } \\
\text { experiences of } \\
\text { HVW }(n=259)\end{array}$ & $\begin{array}{c}\text { Total } \\
\text { wives } \\
(n=360)\end{array}$ \\
\hline & n (\%) & n (\%) & n (\%) & n (\%) & n (\%) & n (\%) \\
\hline \multicolumn{7}{|l|}{ Wife's age } \\
\hline $18-25$ years & $33(9.2)$ & $22(6.1)$ & $19(5.3)$ & $23(6.4)$ & $51(14.2)$ & $74(20.6)$ \\
\hline $26-33$ & $57(15.8)$ & $38(10.6)$ & $18(5.0)$ & $40(11.1)$ & $73(20.3)$ & $113(31.4)$ \\
\hline $34-41$ & $12(3.3)$ & $22(6.2)$ & $30(8.3)$ & $14(3.9)$ & $50(13.9)$ & $64(17.8)$ \\
\hline $42-49$ & $12(3.3)$ & $32(8.9)$ & $30(8.3)$ & $15(4.2)$ & $59(16.3)$ & $74(20.5)$ \\
\hline$\geq 50$ years & $6(1.7)$ & $6(1.7)$ & $23(6.4)$ & $9(2.5)$ & $26(7.2)$ & $35(9.7)$ \\
\hline \multicolumn{7}{|l|}{ Husband's age } \\
\hline 25-31 years & $48(13.3)$ & $38(10.6)$ & $10(2.8)$ & $37(10.3)$ & $59(16.4)$ & $96(26.7)$ \\
\hline $32-38$ & $30(8.3)$ & $28(7.8)$ & $22(6.1)$ & $22(6.1)$ & $58(16.1)$ & $80(22.2)$ \\
\hline $39-45$ & $30(8.3)$ & $22(6.2)$ & $30(8.3)$ & $19(5.3)$ & $63(17.5)$ & $82(22.8)$ \\
\hline $46-52$ & $6(1.7)$ & $24(6.7)$ & 12(3.3) & $9(2.5)$ & $33(9.2)$ & $42(11.7)$ \\
\hline$\geq 53$ years & $6(1.7)$ & $8(2.2)$ & $46(12.7)$ & 14(3.9) & $46(12.7)$ & $60(16.6)$ \\
\hline \multicolumn{7}{|l|}{ Wife's age at marriage } \\
\hline $13-18$ years & $30(8.3)$ & $36(10.0)$ & $65(18.1)$ & $19(5.3)$ & $112(31.1)$ & $131(36.4)$ \\
\hline $19-24$ & $90(25.0)$ & $68(18.9)$ & $48(13.3)$ & $74(20.6)$ & $132(36.6)$ & $206(57.2)$ \\
\hline $25-30$ years & $\mathrm{Nil}$ & $16(4.5)$ & $7(1.9)$ & $8(2.2)$ & $15(4.2)$ & $23(6.4)$ \\
\hline \multicolumn{7}{|l|}{ Husband's age at marriage } \\
\hline $15-20$ years & $18(5.0)$ & $30(8.3)$ & 14(3.9) & $15(4.2)$ & $47(13.0)$ & $62(17.2)$ \\
\hline $21-26$ & $72(20.0)$ & $68(18.9)$ & $64(17.8)$ & $65(181)$ & $139(38.6)$ & $204(56.7)$ \\
\hline 27-34 years & $30(8.3)$ & $22(6.1)$ & $42(11.7)$ & $21(5.8)$ & $73(20.3)$ & $94(26.1)$ \\
\hline \multicolumn{7}{|l|}{ Wife's education } \\
\hline Illiterate & & & $27(7.5)$ & $19(5.3)$ & $89(24.7)$ & $108(30.0)$ \\
\hline Can only & $\begin{array}{l}55(15.3) \\
18(50)\end{array}$ & $\begin{array}{c}26(7.2) \\
2(6)\end{array}$ & $\begin{array}{l}27(7.5) \\
10(28)\end{array}$ & $15(4.2)$ & $15(4.2)$ & $30(8.3)$ \\
\hline read/write & $6(17)$ & $16(4.4)$ & $31(8.6)$ & $11(3.01)$ & $42(11.7)$ & $53(14.7)$ \\
\hline Under secondary & $32(89)$ & $56(15.6)$ & $31(8.6)$ & $28(7.8)$ & $91(25.3)$ & $119(33.1)$ \\
\hline Secondary & $9(2.5)$ & $20(5.6)$ & $\begin{array}{l}31(0.6) \\
21(5.8)\end{array}$ & $28(7.8)$ & $22(6.1)$ & $50(13.9)$ \\
\hline \multirow{2}{*}{\multicolumn{7}{|c|}{ Husband's education }} \\
\hline & & & & & & \\
\hline Illiterate & $46(12.7)$ & $24(6.6)$ & $15(4.3)$ & $15(4.2)$ & $70(19.4)$ & $85(23.6)$ \\
\hline Can only & $21(15.8)$ & $6(1.7)$ & $25(6.9)$ & $21(5.8)$ & $31(8.6)$ & $52(14.4)$ \\
\hline $\mathrm{read} / \mathrm{write}$ & $15(4.2)$ & $12(3.3)$ & $31(8.6)$ & $9(2.5)$ & $49(13.6)$ & $58(16.1)$ \\
\hline Under secondary & $20(5.6)$ & $50(13.9)$ & $37(10.2)$ & $22(6.1)$ & $85(23.6)$ & $107(29.7)$ \\
\hline Secondary & $\begin{array}{l}20(5.0) \\
18(5.0)\end{array}$ & $28(7.8)$ & $12(3.3)$ & $34(9.4)$ & $24(6.7)$ & $5(16.1)$ \\
\hline Upper secondary & & & & & & \\
\hline \multicolumn{7}{|l|}{ Wife's income (EPM) } \\
\hline Nil & $38(10.6)$ & $86(23.9)$ & $70(19.4)$ & $47(13.1)$ & $147(40.8)$ & $194(53.9)$ \\
\hline $100-200$ & $22(6.1)$ & $8(2.2)$ & $15(4.2)$ & $14(3.9)$ & $31(8.6)$ & $45(12.5)$ \\
\hline $201-300$ & $30(8.3)$ & $8(2.2)$ & $6(1.7)$ & $14(3.9)$ & $30(8.3)$ & $44(12.2)$ \\
\hline $301-400$ & $30(8.3)$ & $6(1.7)$ & $3(.8)$ & $14(3.9)$ & $25(6.9)$ & $39(10.8)$ \\
\hline$\geq 401$ & Nil & $12(3.3)$ & $26(7.3)$ & $12(3.3)$ & $26(7.3)$ & $38(10.6)$ \\
\hline \multicolumn{7}{|l|}{ Husband's income (EPM) } \\
\hline $100-500$ & $76(21.1)$ & $50(13.9)$ & $22(6.1)$ & $47(13.0)$ & $101(28.1)$ & $148(41.1)$ \\
\hline $501-900$ & $40(11.1)$ & $53(14.7)$ & $33(9.2)$ & $33(9.2)$ & $93(25.8)$ & $126(35.0)$ \\
\hline $901-1300$ & $4(1.1)$ & $11(3.1)$ & $24(6.7)$ & $9(2.5)$ & $30(8.4)$ & $39(10.9)$ \\
\hline $1301-1700$ & $\mathrm{Nil}$ & $4(1.1)$ & $12(3.3)$ & $8(2.2)$ & $8(2.2)$ & $16(4.4)$ \\
\hline$\geq 1701$ & $\mathrm{Nil}$ & $2(.6)$ & $29(8.0)$ & $4(1.1)$ & $27(7.5)$ & $31(8.6)$ \\
\hline
\end{tabular}


Table 2 (Continued)

\begin{tabular}{|c|c|c|c|c|c|c|}
\hline \multirow{3}{*}{ Characteristics } & \multicolumn{3}{|c|}{ Developmental level of Village } & \multicolumn{3}{|c|}{ Total HVW (Any form) } \\
\hline & $\begin{array}{c}\text { Backward } \\
\text { village } \\
(n=120)\end{array}$ & $\begin{array}{c}\text { Transitional } \\
\text { village } \\
(n=120)\end{array}$ & $\begin{array}{l}\text { Advanced } \\
\text { village } \\
(n=120)\end{array}$ & $\begin{array}{c}\text { wives } \\
\text { without } \\
\text { lifetime } \\
\text { experiences } \\
\text { of HVW } \\
(\mathrm{n}=101) \\
\end{array}$ & $\begin{array}{l}\text { wives with } \\
\text { lifetime } \\
\text { experiences } \\
\text { of HVW } \\
(n=259)\end{array}$ & $\begin{array}{c}\text { Total } \\
\text { wives } \\
(n=360)\end{array}$ \\
\hline & $n(\%)$ & n (\%) & n (\%) & n (\%) & $n(\%)$ & $n(\%)$ \\
\hline \multicolumn{7}{|l|}{ Family income (EPM) } \\
\hline $166-666$ & $37(10.2)$ & $44(12.2)$ & $22(6.1)$ & $30(8.3)$ & $73(20.2)$ & $103(28.5)$ \\
\hline $667-1266$ & $53(14.7)$ & $64(17.8)$ & $18(5.0)$ & $45(12.5)$ & $90(25.0)$ & $135(37.5)$ \\
\hline $1267-1866$ & $24(6.6)$ & $6(1.7)$ & $24(6.6)$ & $14(3.9)$ & $40(11.1)$ & $54(15.0)$ \\
\hline $1867-2466$ & $6(1.7)$ & $6(1.7)$ & $15(4.2)$ & $8(2.2)$ & $19(5.4)$ & $27(7.6)$ \\
\hline$\geq 2467$ & Nil & $\mathrm{Nil}$ & $41(11.4)$ & $4(1.1)$ & $37(10.3)$ & $41(11.4)$ \\
\hline \multicolumn{7}{|l|}{ Quality of dwelling } \\
\hline Bad & $39(10.8)$ & $14(3.9)$ & $24(6.7)$ & $18(5.0)$ & $59(16.4)$ & $77(21.4)$ \\
\hline Middle & $56(15.6)$ & $49(13.6)$ & $62(17.2)$ & $36(10.0)$ & $131(36.4)$ & $167(64.4)$ \\
\hline Good & $25(6.9)$ & $57(15.9)$ & $34(9.4)$ & $47(13.0)$ & 69(19.2) & $116(32.2)$ \\
\hline \multicolumn{7}{|l|}{$\begin{array}{l}\text { Degree of family income } \\
\text { adequate for living }\end{array}$} \\
\hline Not enough & $3(.8)$ & $14(3.9)$ & $34(9.5)$ & $9(2.5)$ & $42(11.7)$ & $51(14.2)$ \\
\hline Barely enough & $29(8.1)$ & $46(12.8)$ & $46(12.8)$ & $29(8.1)$ & $92(25.6)$ & $121(33.7)$ \\
\hline Enough & $88(24.4)$ & $44(12.2)$ & $31(8.6)$ & $51(14.1)$ & $112(31.1)$ & $163(45.2)$ \\
\hline Very enough & $\mathrm{Nil}$ & $16(4.4)$ & $9(2.5)$ & $12(3.3)$ & $13(3.6)$ & $25(6.9)$ \\
\hline \multicolumn{7}{|l|}{ Length of cohabitation } \\
\hline$\leq 6$ years & $42(11.7)$ & $34(9.4)$ & $16(4.4)$ & $35(9.7)$ & $57(15.8)$ & $92(25.5)$ \\
\hline $7-12$ & $42(11.7)$ & $24(6.6)$ & $15(4.2)$ & $27(7.5)$ & $54(15.0)$ & $81(22.5)$ \\
\hline $13-18$ & $12(3.3)$ & $24(6.7)$ & $15(4.2)$ & $9(2.5)$ & $42(11.7)$ & $51(14.2)$ \\
\hline $19-24$ & $15(4.2)$ & $14(3.9)$ & $31(8.6)$ & $17(4.7)$ & $43(11.9)$ & $60(16.7)$ \\
\hline$\geq 25$ years & $9(2.5)$ & $24(6.7)$ & $43(11.9)$ & $13(3.6)$ & $63(17.5)$ & $76(21.1)$ \\
\hline \multicolumn{7}{|l|}{ No. of living children } \\
\hline Non child & $3(.8)$ & $2(.6)$ & $10(2.8)$ & $3(.8)$ & $12(3.4)$ & $15(4.2)$ \\
\hline $1-2$ & $74(20.6)$ & $25(6.9)$ & $33(9.2)$ & $46(12.8)$ & $86(23.9)$ & $132(36.7)$ \\
\hline$\geq 3$ children & $43(11.9)$ & $25.8)$ & $77(21.4)$ & $52(14.4)$ & $161(44.7)$ & $213(59.1)$ \\
\hline \multicolumn{7}{|l|}{ Social level of neighbors } \\
\hline Low level & $27(7.5)$ & Nil & $4(1.1)$ & $13(3.6)$ & $18(5.0)$ & $31(8.6)$ \\
\hline Middle level & $80(22.2)$ & $114(31.7)$ & $107(29.7)$ & $73(20.3)$ & $228(63.3)$ & $301(83.6)$ \\
\hline High level & $13(3.6)$ & $6(1.7)$ & $9(2.5)$ & $15(4.2)$ & $13(3.6)$ & $\begin{array}{l}28(7.8) \\
\end{array}$ \\
\hline
\end{tabular}

\section{Results}

\subsection{Characteristics of respondents exposed to HVW during the last 12 months}

Table 2 shows that the percentage of wives exposed to any form of HVW during the last 12 months was $71.9 \%$. The largest percentage of them were aged 26-33 years old, married at 19-24 years old, attained secondary education, had no personal income, having middle quality of dwelling, having adequate enough of family income for living, with 25 years or more length of cohabitation, having 3 children or more, and living in middle social level of neighborhood.

\subsection{Prevalence of HVW committed against wives}

\subsubsection{Prevalence of HVW committed against wives by forms}

The results from Table 3 and Fig. 2 overall, showed that $66.7 \%$ (240 out of 360) of the wives in this study clearly reported of having experienced psychological HVW alone in the past year prior the 
survey, $172(47.8 \%)$ of them reported physical HVW alone, and 155 $(43.1 \%)$ of them suffered sexual HVW alone. A considerable overlap was found among psychological, physical and sexual HVW, 45.3\%\% had experienced both psychological or physical HVW, $40 \%$ had experienced either psychological or sexual HVW, 30.2\% had experienced either physical or sexual HVW, $29.4 \%$ had experienced all forms of HVW. Wives suffered from more than one type of violence can also be seen in Fig. 2.

Table 3. Percentage of HVW Forms experienced by respondents during the Past Year

\begin{tabular}{|lcc|}
\hline \multicolumn{1}{|c|}{ Forms of HVW } & $\begin{array}{c}\text { All married women } \\
(\mathbf{n = 3 6 0 )} \%\end{array}$ & $\begin{array}{c}\text { Married women abused by HVW } \\
(\mathbf{n = 2 5 9 )} \%\end{array}$ \\
\hline Psychological HVW alone & 66.7 & 92.7 \\
Physical HVW alone & 47.8 & 66.4 \\
Sexual HVW alone & 43.1 & 59.8 \\
Psychological or Physical HVW & 45.3 & 62.9 \\
Psychological or Sexual HVW & 40.0 & 55.6 \\
Physical or Sexual HVW & 30.8 & 42.9 \\
All forms of HVW & 29.4 & 39.8 \\
\hline
\end{tabular}

Percentages do not add up to 100 due to multiple responses

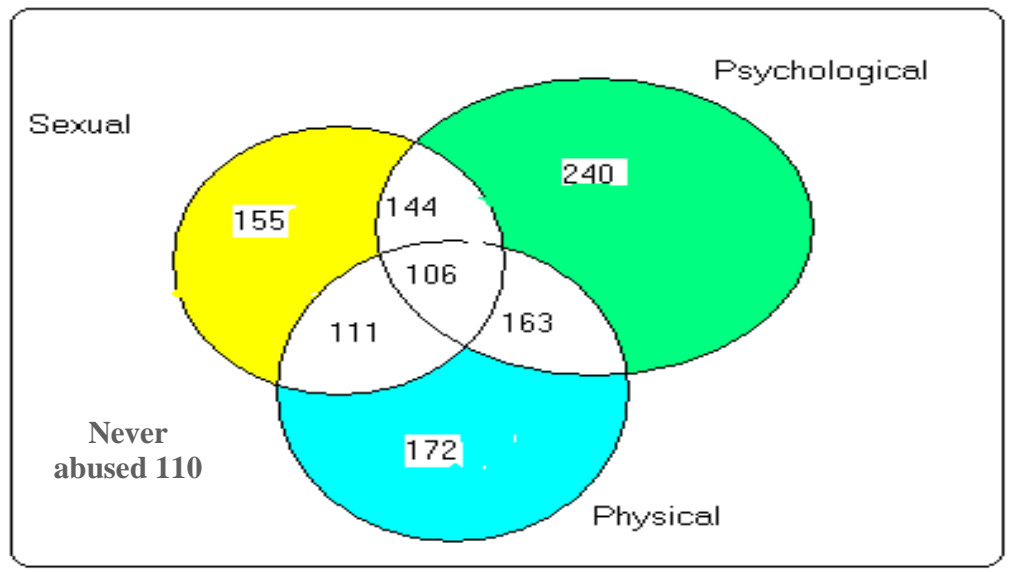

Fig. 2. The overlap between psychological, physical and sexual HVW experienced by 259 out of 360 respondents during past 12 months

\subsubsection{Prevalence of HVW committed against wives by acts}

Table 4 shows that the sexual HVW was ranked the first of frequency of HVW, followed by psychological HVW, finally physical HVW. The average weighted of these three groups of HVW were 201, 162.5 , and 116.2, respectively.

The main HVW acts were ranked slapped or twisted arm, followed by forced to have sex in an unwanted time, humiliated, insulted, prevented from talking, gave angry stares or looks, pushed, labeling with demeaning terms (e.g., "crazy", "animal"), prevented from health 
care, unwanted or forced touching, respectively. The total weighted scores of these types were 504, 479, 379, 308, 243, 227, 224, 223, and 212 , respectively.

Table 4 Frequencies of HVW acts reported by all respondents rural wives $(n=360)$

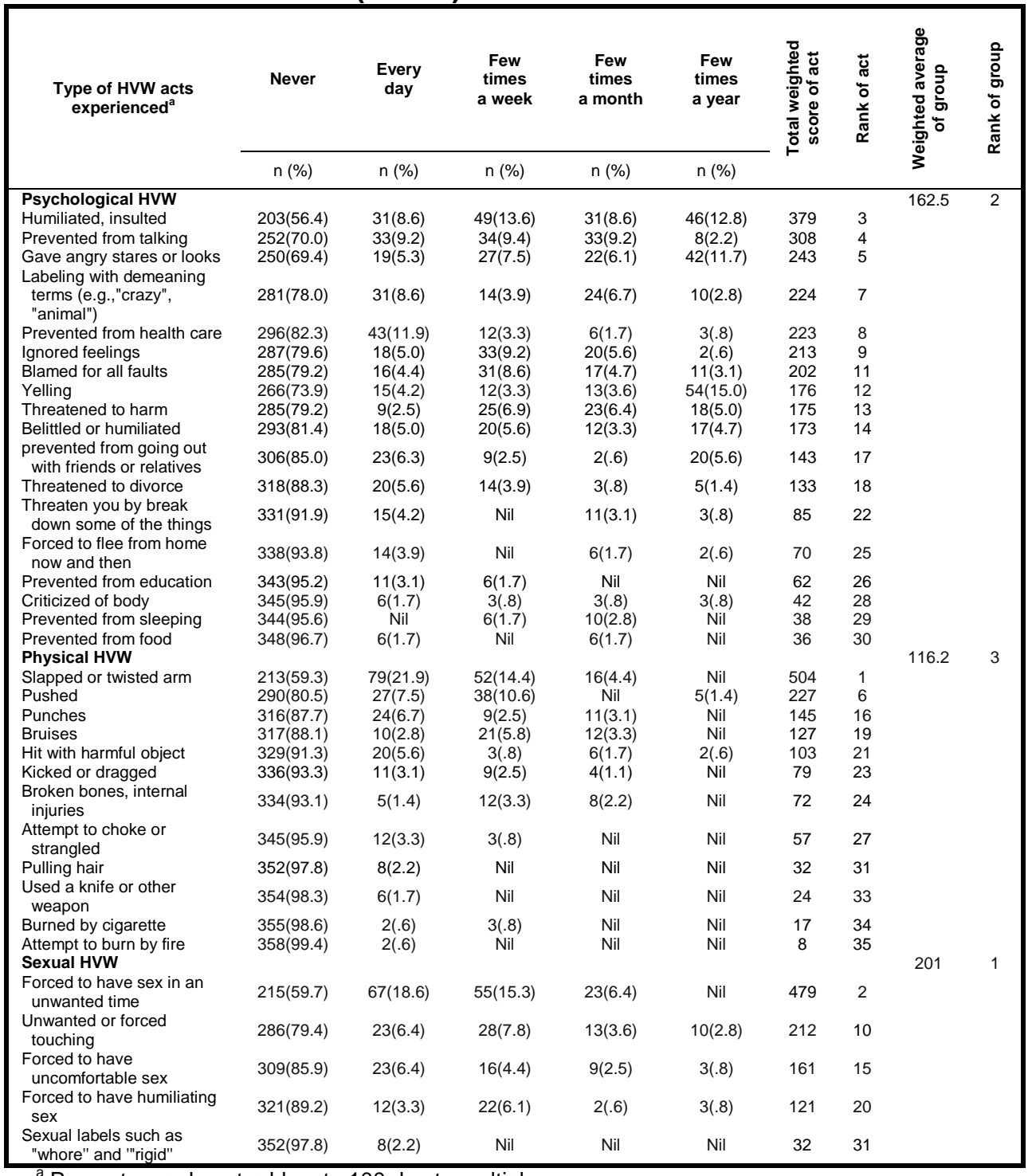

${ }^{a}$ Percentages do not add up to 100 due to multiple responses

\subsection{Reasons of HVW}


Table 5 indicates the frequencies of reasons why HVW occurred (259 out of 360) in the past year preceding the study. The main indicated reasons include: the desire to visit parents, followed by husband influenced by friends or relatives, without reason/l could not understand, difference of view, jealousy, he reflects distress in his work, economic reason, husband's own social problems, not to cook the food he likes, and going out to walk, respectively. The total weighted scores of these reasons were 227, 212, 211, 194, 190, 180, $170,159,122$, and 107 respectively.

Table 5. Frequencies of reasons of HVW reported by abused wives $(n=259)$

\begin{tabular}{|c|c|c|c|c|c|c|}
\hline \multirow[t]{2}{*}{ Reasons $^{\mathrm{a}}$} & None & Rarely & Sometimes & Frequently & \multirow{2}{*}{ 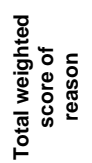 } & \multirow[t]{2}{*}{ 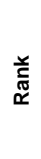 } \\
\hline & n (\%) & n (\%) & n (\%) & n (\%) & & \\
\hline The desire to visit your parents & $137(52.8)$ & $57(22.0)$ & $31(11.9)$ & $36(13.9)$ & 227 & 1 \\
\hline Husband influenced by friends or relatives & $139(53.6)$ & $64(24.7)$ & $26(10.0)$ & $32(12.4)$ & 212 & 2 \\
\hline Without reason/I could not understand & $159(61.4)$ & $36(13.9)$ & $23(8.9)$ & $43(16.6)$ & 211 & 3 \\
\hline Difference of view & $158(60.0)$ & $41(15.8)$ & $33(12.7)$ & $29(11.2)$ & 194 & 4 \\
\hline Jealousy & $150(57.9)$ & $54(20.8)$ & $35(13.5)$ & $22(8.5)$ & 190 & 5 \\
\hline He reflects distress in his work & $146(56.4)$ & $52(20.1)$ & $52(20.1)$ & $8(3.1)$ & 180 & 6 \\
\hline Economic reason & $164(63.3)$ & $40(15.4)$ & $41(15.8)$ & $16(6.1)$ & 170 & 7 \\
\hline Husband's own social problems & $170(65.6)$ & $37(14.2)$ & $40(15.4)$ & $14(5.4)$ & 159 & 8 \\
\hline Not to cook the food he like & $195(75.2)$ & $31(11.9)$ & $14(5.4)$ & $21(8.1)$ & 122 & 9 \\
\hline Going out to walk & $205(79.1)$ & $25(9.6)$ & $11(4.2)$ & $20(7.7)$ & 107 & 10 \\
\hline Failure to fulfill husband's sexual will & $195(75.2)$ & $51(19.6)$ & $9(3.4)$ & $6(2.3)$ & 87 & 11 \\
\hline Husband's abuse of drugs & $229(88.4)$ & $5(1.9)$ & $12(4.6)$ & $15(5.8)$ & 74 & 12 \\
\hline Failure to fulfill domestic duties & $223(86.1)$ & $14(5.4)$ & $21(8.1)$ & $3(1.1)$ & 65 & 13 \\
\hline \multirow{3}{*}{$\begin{array}{l}\text { Husband's getting married against own will } \\
\text { Behaviors contrary to traditional perceptions } \\
\quad \text { of honor } \\
\text { Going out without permission }\end{array}$} & $236(91.1)$ & $7(2.7)$ & $3(1.1)$ & $15(5.7)$ & 58 & 14 \\
\hline & $225(86.8)$ & $22(8.4)$ & $8(3.0)$ & $6(2.3)$ & 56 & 15 \\
\hline & $234(90.3)$ & $9(3.4)$ & $15(5.7)$ & $3(1.1)$ & 48 & 16 \\
\hline $\begin{array}{l}\text { Not convinced the man to the type and the } \\
\text { way you dress }\end{array}$ & $227(87.6)$ & $30(11.5)$ & Nil & $4(1.5)$ & 42 & 17 \\
\hline Lack of love and respect & $240(92.6)$ & $10(3.8)$ & $8(3.0)$ & $3(1.1)$ & 35 & 18 \\
\hline Wife want to work outside home & $239(92.2)$ & $19(7.3)$ & $\mathrm{Nil}$ & $3(1.1)$ & 28 & 19 \\
\hline
\end{tabular}

${ }^{a}$ Percentages do not add up to 100 due to multiple responses.

\subsection{Consequences of HVW}

Table 6 shows that the main frequencies of consequences of HVW reported by abused wives during the prior year were : irritability, followed by anxiety, fearing, eating disorders, sleeping disorders, frustration, isolation, insecurity feeling, depression, injury, low selfesteem, and physical inactivity, respectively. The total weighted score of these consequences of HVW were 357, 342, 339, 333, 278, 258, $256,253,250,170,157$, and 125 , respectively.

Table 6. Frequencies of consequences of HVW reported by abused wives $(n=259)$

\begin{tabular}{|c|c|c|c|c|c|c|c|}
\hline \multirow{3}{*}{ Consequences $^{a}$} & \multirow{3}{*}{$\begin{array}{c}\begin{array}{c}\text { Never } \\
\text { happened }\end{array} \\
\mathrm{n}(\%)\end{array}$} & \multicolumn{4}{|c|}{ Happened } & \multirow{3}{*}{$\begin{array}{c}\text { Total } \\
\text { weighted } \\
\text { score of } \\
\text { consequenc } \\
\text { e }\end{array}$} & \multirow{3}{*}{ Rank } \\
\hline & & Rarely & $\begin{array}{c}\text { Sometime } \\
\mathbf{s}\end{array}$ & Often & Always & & \\
\hline & & n (\%) & n (\%) & n (\%) & $\mathrm{n}(\%)$ & & \\
\hline Irritability & $187(51.9)$ & $59(16.4)$ & $57(15.8)$ & $44(12.2)$ & $13(3.6)$ & 357 & 1 \\
\hline Anxiety & $190(52.8)$ & $59(16.4)$ & $68(18.9)$ & $25(6.9)$ & $18(5.0)$ & 342 & 2 \\
\hline Fearing & $194(53.9)$ & $63(17.5)$ & $48(13.3)$ & $40(11.1)$ & $15(4.2)$ & 339 & 3 \\
\hline
\end{tabular}




\begin{tabular}{lccccccc}
\hline Eating disorders & $189(52.5)$ & $64(17.8)$ & $55(15.6)$ & $45(12.5)$ & $6(1.7)$ & 333 & 4 \\
Sleeping disorders & $219(6.8)$ & $57(15.8)$ & $38(10.6)$ & $39(10.8)$ & $7(1.9)$ & 278 & 5 \\
Frustration & $248(68.9)$ & $34(9.4)$ & $28(7.8)$ & $32(8.9)$ & $18(5.0)$ & 258 & 6 \\
Isolation & $239(66.4)$ & $48(13.3)$ & $31(8.6)$ & $22(6.1)$ & $20(5.6)$ & 256 & 7 \\
Insecurity feeling & $226(62.8)$ & $63(17.5)$ & $35(9.7)$ & $24(6.7)$ & $12(3.3)$ & 253 & 8 \\
Depression & $244(67.8)$ & $42(11.7)$ & $28(7.8)$ & $32(8.9)$ & $14(3.9)$ & 250 & 9 \\
Injury & $249(69.2)$ & $52(14.4)$ & $43(11.9)$ & $8(2.2)$ & $2(2.2)$ & 170 & 10 \\
Low self-esteem & $273(75.8)$ & $36(10.0)$ & $32(8.9)$ & $19(5.3)$ & Nil & 157 & 11 \\
Physical inactivity & $295(81.9)$ & $23(6.4)$ & $26(7.2)$ & $14(3.9)$ & $2(.6)$ & 125 & 12 \\
\hline
\end{tabular}

Percentages do not add up to 100 due to multiple responses

\subsection{Determinants of HVW}

To assess the effects of social, demographic, economic and cultural variables (mentioned in Table 2) taken together, on the prevalence of HVW. The prevalence of whether or not the wives had ever experienced HVW any time is the dependent variable. The approach was to fit a multinomial logistic regression model with a large pool of candidate explanatory variables. First, the model with all explanatory variables was fitted and then certain explanatory variables were removed, which were distorting the model. Finally, This study maintained the following variables in the analysis: wife's age, husband's age, wife's age at marriage, husband's age at marriage, wife's education, husband's education, wife's income EPM, husband's income EPM, family income EPM, quality of dwelling, degree of family income adequate for living, Length of cohabitation, number of living children, social level of neighbors, and developmental level of village. Table 7 reflects the results of the logistic regression analysis, based on answers given by 630 of the interviewed married women. A total of 15 variables including two dummy variables are used as independent variables. The abbreviation "rc" in the table stands for the "reference category".

Looking at the odds ratios, we can observe that if a husband is married at 21-26 years old, this would decrease HVW against their wives compared to those who married at 15-20 category. Likewise, when husbands get 1701 EPM personal income and more, this will decrease HVW against their wives, compared to those who get 100500 EPM. Also, when cohabitation length reaches 25 years or more, the HVW against woman will decrease when compared to 6 years or less. As compared to the backward village the advanced village is less than other villages regarding HVW against women. The odds ratios are lower than 1.

In contrast to the assumptions, this study found that when wives can only read/write, this will increase HVW against them, compared to those who illiterate. Also, husband's education is positively associated with HVW.

Also, wife's income is positively associated with HVW. As the income category rises, the level of positive significance decrease; in other words the significance level falls from $1 \%$ levels to $5 \%$. The odds ratios are above 1 for the higher income categories of 100-200 EPM 
(which is significant at $1 \%$ confidence level), and 301-400 EPM (which is significant at $5 \%$ confidence level), indicating that the incidence of violence is much greater as compared to housewife or woman who don't work; the category of nil EPM being the reference category. This study has also found that husband's education is positively associated with HVW. As the educational level rises, the level of positive significance also rises to the secondary category then decrease at the upper secondary category (which are significant at $5 \%$ ) compared to 100-500 EPM category.

Table 7. Determinants of HVW from Multinomial logistic regression analysis

\begin{tabular}{|c|c|c|c|c|c|c|}
\hline \multirow{2}{*}{$\begin{array}{l}\text { Variables in the } \\
\text { equation }\end{array}$} & \multirow{2}{*}{ Categories } & \multirow{2}{*}{$B$} & \multirow{2}{*}{ Sig. } & \multirow{2}{*}{$\begin{array}{l}\text { Odds ratios } \\
\text { EXP(B) }\end{array}$} & \multicolumn{2}{|c|}{$95.0 \%$ C.I. for $\operatorname{EXP}(\mathrm{B})$} \\
\hline & & & & & Lower & Upper \\
\hline \multirow[t]{5}{*}{ Wife's age } & $18-25$ years $(\mathrm{rc})$ & & & 1.0 & & \\
\hline & $26-33$ & .576 & .825 & 1.779 & .011 & 289.525 \\
\hline & $34-41$ & -.340 & .877 & .712 & .010 & 52.527 \\
\hline & $42-49$ & 3.334 & .055 & 28.041 & .934 & 841.431 \\
\hline & 50 years or more & 1.151 & .370 & 3.161 & .256 & 39.098 \\
\hline \multirow[t]{5}{*}{ Husband's age } & $25-31$ years $(\mathrm{rc})$ & & & 1.0 & & \\
\hline & $32-38$ & 1.894 & .312 & 6.648 & .170 & 260.693 \\
\hline & $39-45$ & 1.164 & .485 & 3.216 & .121 & 85.223 \\
\hline & $46-52$ & 1.727 & .250 & 5.623 & .296 & 106.748 \\
\hline & 53 years or more & 1.203 & .389 & 3.329 & .216 & 51.372 \\
\hline \multirow{3}{*}{$\begin{array}{l}\text { Wife's age at } \\
\text { marriage }\end{array}$} & $13-18$ years $(\mathrm{rc})$ & & & 1.0 & & \\
\hline & $19-24$ & -.185 & .900 & .831 & .047 & 14.829 \\
\hline & $25-30$ years & -1.252 & .297 & .286 & .027 & 3.004 \\
\hline \multirow{3}{*}{$\begin{array}{l}\text { Husband's age at } \\
\text { marriage }\end{array}$} & $15-20$ years $(\mathrm{rc})$ & & & 1.0 & & \\
\hline & $21-26$ & -3.266 & $.003^{\star \star}$ & .038 & .005 & .320 \\
\hline & $27-34$ years & -1.988 & $.013^{*}$ & .137 & .029 & .656 \\
\hline \multirow[t]{5}{*}{ Wife's education } & Illiterate (rc) & & & 1.0 & & \\
\hline & Can only read/write & 2.399 & $.050^{*}$ & 11.014 & 1.004 & 120.846 \\
\hline & Under secondary & -.337 & .771 & .714 & .074 & 6.918 \\
\hline & Secondary & -.309 & .782 & .782 & .082 & 6.545 \\
\hline & Upper secondary & .822 & .372 & .372 & .372 & 13.824 \\
\hline \multirow{5}{*}{$\begin{array}{l}\text { Husband's } \\
\text { education }\end{array}$} & Illiterate (rc) & & & 1.0 & & \\
\hline & Can only read/write & 2.259 & $.016^{*}$ & 9.574 & 1.526 & 60.082 \\
\hline & Under secondary & -.928 & .369 & .395 & .052 & 2.992 \\
\hline & Secondary & 2.470 & $.013^{*}$ & 11.823 & 1.672 & 83.606 \\
\hline & Upper secondary & 2.006 & $.020^{*}$ & 7.434 & 1.380 & 40.048 \\
\hline \multirow{5}{*}{$\begin{array}{l}\text { Wife's income } \\
\text { (EPM) }\end{array}$} & Nil (rc) & & & 1.0 & & \\
\hline & $100-200$ & 3.099 & $.002^{\star *}$ & 22.170 & 3.014 & 163.074 \\
\hline & $201-300$ & .753 & .496 & 2.123 & .243 & 18.525 \\
\hline & $301-400$ & 2.510 & $.032^{*}$ & 12.305 & 1.243 & 121.836 \\
\hline & 401 or more & 1.388 & .241 & 4.007 & .934 & 40.702 \\
\hline \multirow{5}{*}{$\begin{array}{l}\text { Husband's income } \\
\text { (EPM) }\end{array}$} & $100-500(\mathrm{rc})$ & & & 1.0 & & \\
\hline & $501-900$ & -3.297 & .108 & .037 & .001 & 2.062 \\
\hline & $901-1300$ & -2.421 & .220 & .089 & .002 & 4.267 \\
\hline & $1301-1700$ & -3.759 & .073 & .023 & .000 & 1.417 \\
\hline & 1701 or more & -8.387 & $.001^{* *}$ & .000 & .000 & .036 \\
\hline Family income & $166-666(\mathrm{rc})$ & & & 1.0 & & \\
\hline \multirow{4}{*}{ (EPM) } & $667-1266$ & -.757 & .734 & .169 & .006 & 36.733 \\
\hline & $1267-1866$ & -.913 & .670 & .401 & .006 & 26.863 \\
\hline & $1867-2466$ & .584 & .793 & 1.793 & .023 & 140.291 \\
\hline & 2467 or more & -1.620 & .438 & .198 & .003 & 11.897 \\
\hline \multirow[t]{3}{*}{ Quality of dwelling } & $\mathrm{Bad}(\mathrm{rc})$ & & & 1.0 & & \\
\hline & Middle & 1.156 & .121 & 3.178 & .736 & 13.729 \\
\hline & Good & .587 & .343 & 1.798 & .534 & 6.051 \\
\hline Degree of family & Not Enough (rc) & & & 1.0 & & \\
\hline income adequate & Barely enough & .783 & .528 & 2.188 & .192 & 24.877 \\
\hline for living & Enough & 2.473 & $.023^{*}$ & 11.856 & 1.408 & 99.821 \\
\hline & Very enough & .743 & .447 & 2.102 & .310 & 14.243 \\
\hline Length of & 6 years or less ( $(\mathrm{rc})$ & & & 1.0 & & \\
\hline cohabitation & $7-12$ & -2.127 & .449 & .119 & .000 & 29.218 \\
\hline & $13-18$ & -2.822 & .266 & .059 & .000 & 8.598 \\
\hline & $19-24$ & -2.506 & .227 & .082 & .001 & 4.754 \\
\hline & 25 years or more & -4.012 & $.003^{\star \star}$ & .018 & .001 & .262 \\
\hline No. of living & Non child (rc) & & & 1.0 & & \\
\hline children & $1-2$ & .104 & .954 & 1.110 & .032 & 39.102 \\
\hline & 3 children or more & .230 & .723 & 1.258 & .352 & 4.496 \\
\hline Social level of & Low level (rc) & & & 1.0 & & \\
\hline neighbors & Middle level & -.313 & .762 & .731 & .096 & 5.559 \\
\hline
\end{tabular}




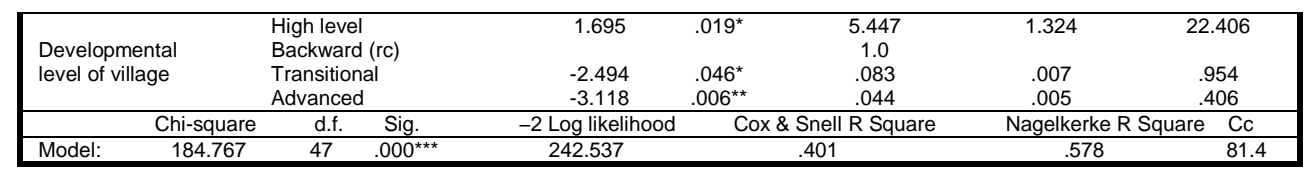

rc: Reference category. Cc: Correctly classified. $\quad * p \leq 0.05 . \quad * * p \leq 0.01 . \quad * * * p \leq 0.001$.

The findings have also found that wives who live next to neighbors had high social level are likely to be more abused with HVW compared with low level. Likewise, wives who reported that their family income adequate enough for living are more likely be abused by HVW compared also positively associated with HVW.

Unexpected findings obtained in this study were the wife's age, husband's age, wife's age at marriage, family income, quality of dwelling, and number of living children does not associated with HVW.

\section{Discussion and Conclusion}

This study investigated the relationships between certain social, demographic, economic and cultural variables and HVW based on a sample survey of three villages with varying developmental levels in the rural areas of Sharkia governorate in Egypt.

There are some interesting results as well, which might be a reflection of the country's characteristics and different developmental levels. The Mediterranean culture and the Islamic tradition have impacts the culture (Muftuler-Bac, 1999).

The most important findings from this study confirm that HVW against married women is highly prevalent. It is a common problem that cannot be ignored in the rural areas of Sharkia governorate of Egypt. The vast majority (71.9\%) of women reported different types of HVW in the last 12 months. This rate of abuse is consistent with the recent studies conducted in Egypt (Diop-Sidibe et al. 2006; Seedhom 2011). Regionally, a study from Jordan reported that $87 \%$ of women reported different types of IPV against them during the past year ( $\mathrm{Al}$ Nsour and Khawaja, 2009).

The prevalence of HVW by form against rural Egyptian wives in the year prior to the sample survey is high. The most common forms of HVW were psychological HVW alone (66.7\%), followed by physical HVW alone (47.8\%), and sexual HVW alone (43.1\%). A considerable overlap was found among psychological, physical and sexual HVW against wives, $29.4 \%$ of women reporting all three kinds of HVW. These frequencies are generally consistent with a study conducted in two rural areas of Bangladesh (Koenig et al., 2003), in a recent study in the rural of Bangladesh Naved et al. (2006) found $42 \%$ of women suffered of IPV in lifetime and past 12 months. In Nicaragua, among ever-married women 52\% reported having experienced physical partner violence at some point in their lives (Ellsberg et al., 2000). 
Regarding the HVW prevalence by acts in this study, the main ranking of HVW acts against women were slapped or twisted arm, followed by forced to have sex in an unwanted time, humiliated, insulted, prevented from talking, gave angry stares or looks, pushed, labeling with demeaning terms (e.g., "crazy", "animal"), prevented from health care, unwanted or forced touching, respectively. The group acts of sexual HVW was ranked first among forms of HVW, followed by group acts of psychological HVW, finally group acts of physical HVW. Largely, these findings are consistent with finding of a recent study in Egypt (El-Zanaty and Way, 2006).

The findings regarding the reasons of HVW in the past year preceding the sample survey found that the main reasons were: the desire to visit her parents, followed by husband influenced by friends or relatives, without reason/l could not understand, difference of view, jealousy, he reflects distress in his work, economic reason, husband's own social problems, and not to cook the food he like, respectively. Largely, these reasons are in agreement with the results obtained earlier in Egypt (El-Zanaty and Way, 2006; National Council for Women, 2006).

Regarding the consequences of HVW in women were related to health effects, both psychological and physical. The main consequences were ranked as follow: irritability, followed by anxiety, fearing, eating disorders, sleeping disorders, frustration, isolation, insecurity feeling, depression, respectively. These findings are in agreement with the results obtained in a study in a rural district in northern Vietnam (Krantz et al., 2005).

The findings regarding the determinants of HVW discovered that the prevalence of HVW is found to decrease as husbands' age at marriage increases over 20 years. This result is consistent with the finding of a recent study in Turkey (Kocacık et al., 2007). This is due to the fact that young husbands have limited ability to understand the social responsibility of family. They lack the ability to understand the natures of wives and how to deal with them when different situations arise inside the family. How to address them through nonviolence instead of with HVW. The results also found that men with higher incomes have fewer HVW toward their wives.

The length of marriage or the long duration of marriage in the present study affects negatively on IPV. This finding is consistent with the finding of a comparative analysis of the 1995 and 2005 Egypt DHS (Akmatov et al., 2008). This research found that over time a marital relationship can achieve a degree of stability. The stability of the relationship reduces the husband's tendency to resort to violence. After many years of marriage, a woman may learn to cope with HVW. This study also concluded that higher developmental level of village 
led to decrease the prevalence of HVW. This is due to the improvement of community services in advanced village reduce the problems of family and reduce violence between husband and his wife.

In contrast to the assumptions, this study has concluded that when wives can only read/write are more likely to be abused by their husband. Also, the prevalence of HVW is found to increase as husbands' educational level increases. The HVW is due to the educated husbands in Egypt suffered from shortage of adequate personal income, where the salaries are not sufficient to the living needs. Problems between couples will occur due to this reason, this is leading to violence against their wives.

Also, as a wife's personal income rises it increased her likelihood to be abused by her husband. This finding is inconsistent with the finding reached by many researchers, in Egypt (Akmatov et al., 2008), and in Turkey (Kocacık et al., 2007). Empirical Studies found that the fewer income resources a woman has, the less power she has; therefore, she is less likely to leave the abusive relationship (Awang and Hariharan, 2011, p.464). In contrary to these studies, the social and cultural context in the rural areas of Egypt, may influence males to act in a violent manner. This study indicated that husbands often disapproval of wives being attaining higher economic status because they may be more likely to challenge their husbands' authority. The husbands may perceive this as a threat to their authority. This may explain why wives who have no personal income are less likely to face HVW. This finding is consistent with the finding obtained in a study in rural areas of Bangladesh (Bates et al., 2004,p195).

Likewise, this study has concluded that the prevalence of HVW will increase as the family income is just adequate enough for living. This is due to this category of family need to more adequate income.

Also, the HVW is found to be increased social level of neighbors was at high level in this study. This is also consistent with finding obtained recently in Turkey (Kocacık et al., 2007). This is due to the nature of the culture of rural women in Egypt, which are jealous of their neighbors, with a higher social level. Violence may occur from husband against his wife as an outcome of her claim to improve the standard of living of her family as neighbors.

Unlike other studies this study found that wife's age, husband's age, wife's age at marriage, family income, quality of dwelling, or number of living children were not associated with HVW.

\section{Recommendations}

The findings reported here suggest the need for:

(a) IPV should receive more attention from policy makers in Egypt. The for more effective advocacy programmes at all levels. 
(b) Improved counseling and training programmes made available to health care workers, members of women's civil organizations, women's groups, and other officials.

(c) The development of local advocacy groups that educate the public on HVW and offer training on how to address victimization of women by their partners in the rural sectors of Egypt.

(d) Egyptian women should be empowered by the government, NGO and stakeholders to earn better income, especially through generating small incomes independent of their husbands.

(e) Egyptian husbands should be educated on the importance of nonviolence an appropriate language during discussion on all issues with their wives.

(f) Increase awareness of men, women, male and female youth of Islamic religious teachings of the obligations of parents toward their children to ensure their psychological, physical, mental, religious, and cultural well-being to improve their future livelihood. That should be done in all local mosques, women organizations and through the use of mass media.

\section{REFERENCES}

Akmatov, M.K. et al. 2008. Research article: Factors associated with wife beating in Egypt: Analysis of two surveys (1995 and 2005). BMC Women's Health. Available from http://www.biomedcentral.com/14726874/8/15.

Al-Nsour, M.,and Khawaja, M. 2009. Domestic Violence against Women in Jordan: Evidence from Health Clinics. Journal of Family Violence 24, Issue 6, pp569-575.

Amnesty International. 2008. The State of the World's Human Right. International Secretariat, United Kingdom.

Awang, H. and Hariharan, S. 2011. Determinants of Domestic Violence: Evidence from Malaysia. Journal of Family Violence 26, Issue 6, pp459-464.

Bates, L., M.; Schuler, S., R; Islam, F. and Islam, M., K. 2014. Socioeconomic Factors and Processes Associated With Domestic Violence in Rural Bangladesh. International Family Planning Perspectives, 30(4), pp190-199.

Bureau of Sharkia governorate. 2014. Statement of organizations services. Center of Decision Support and Information.

Carrillo R. 2002. Overview of international human rights standards and other agreements and responses of the judicial system to violence against women. International Journal of Gynecology and Obstetrics 78 Suppl, 1, ppS15-S20.

Center for Disease Control and Prevention (CDC). 2004. Sexual violence prevention: beginning the dialogue. Atlanta, GA: Centers for Disease Control and Prevention. 
Cochran, W.G. 1977. Sampling Techniques. John Wiley and Sons. 2ed., U.S.A.

Dahlberg, L.L., and Krug, E.G. 2002. chapter one : Violence - a global public health problem. In Krug, E.G. et al. (Eds.). (2002). World report on violence and health. Geneva: World Health Organization. Available from http://whqlibdoc.who.int/hq/2002/9241545615.pdf.

Diop-Sidibe, N., Campbell, J.C., and Becker, S. 2006. Domestic violence against women in Egypt-wife beating and health outcomes. Social Science \& Medicine 62, pp1260-1277.

Ellsberg M, and Heise L. 2005. Researching Violence Against Women: A Practical Guide for Researchers and Activists. Washington DC, United States: World Health Organization, PATH.

Ellsberg, M., et al. 2000. Candies in hell: women's experiences of violence in Nicaragua. Social Science and Medicine 51, pp5951610.

El-Zanaty, F. and Way, A. 2006. Egypt Demographic and Health Survey 2005. Cairo, Egypt: Ministry of Health and Population, National Population Council, El-Zanaty and Associates, and ORC Macro.

Heise, L. 1998. Violence against women: An integrated, ecological framework. Violence Against Women, Vol. 4, No.3, pp 262-290.

Heise, Lori. L. 2011. What Works to Prevent Partner Violence? An Evidence Overview. Report for the UK Department for International Development.

Heise, L., Ellsberg, M. and Gottemoeller, M. 1999. Ending Violence Against Women. Population Reports, Series L, No. 11. Baltimore, Johns Hopkins University School of Public Health, Population Information Program.

Heise, L.; Ellsberg, M. and Gottmoeller, M. 2002. A global overview of gender-based violence . International Journal of Gynecology and Obstetrics 78, Suppl. 1, ppS5-S1.4

Kocacık, F.; Kutlar, A. and Erselcan, F. 2007. Domestic violence against women: A field study in Turkey. The Social Science Journal 44, pp698-720.

Koenig, M.A., et al. 2003. Women's status and domestic violence in rural Bangladesh: individual- and community level effects. Demography 40, pp 269-288.

Krantz, G., et al. 2005. Intimate partner violence: forms, consequences and preparedness to act as perceived by healthcare staff and district and community leaders in a rural district in northern Vietnam. Public Health 119, pp1048-1055.

Krug, E.G.; Mercy, J.,A.; Dahlberg, L., L. and Zwi, A., B. 2002. The world report on violence and health. Lancet, Vol. 360, Issue 9339,pp 1083-1088.

Ministry of Health and Population [Egypt], El-Zanaty and Associates [Egypt], and ICF International. 2015. Egypt Demographic and Health Survey 2014. Cairo, Egypt and Rockville, Maryland, USA: Ministry of Health and Population and ICF International. 
Muftuler-Bac, M. 1999. Turkish women's predicament. Women's Studies International Forum, Vol 22 (3), pp 303-315.

National Council for Women. 2006. The Final Report of the Field Study: The Multifaceted Development Project for the Advancement of Women in Minya Governorate.

Naved, R.T., Azim, S., Bhuiya, A., and Persson, L.A. 2006. Physical violence by husbands: Magnitude, disclosure and help-seeking behavior of women in Bangladesh. Social Science \& Medicine 62, pp 2917-2929.

Rosche, D. 2014. Close the GaP : HOW TO Eliminate Violence Against Women Beyond 2015. OXFAM, Oxfam GB for Oxfam International, Oxford, OX4 2JY, UK, ISBN 978-1-78077-565-4. www.oxfam.org.

Seedhom, A.D. 2011. Sociodemographic associations of intimate partner violence against women in a rural area, El-Minia governorate, Egypt, (2010). J Public Health. DOI 10.1007/s10389-011-0431-5.

World Health Organization (WHO)/London School of Hygiene and Tropical Medicine. 2010. Preventing intimate partner and sexual violence against women: taking action and generating evidence. Geneva, World Health Organization..
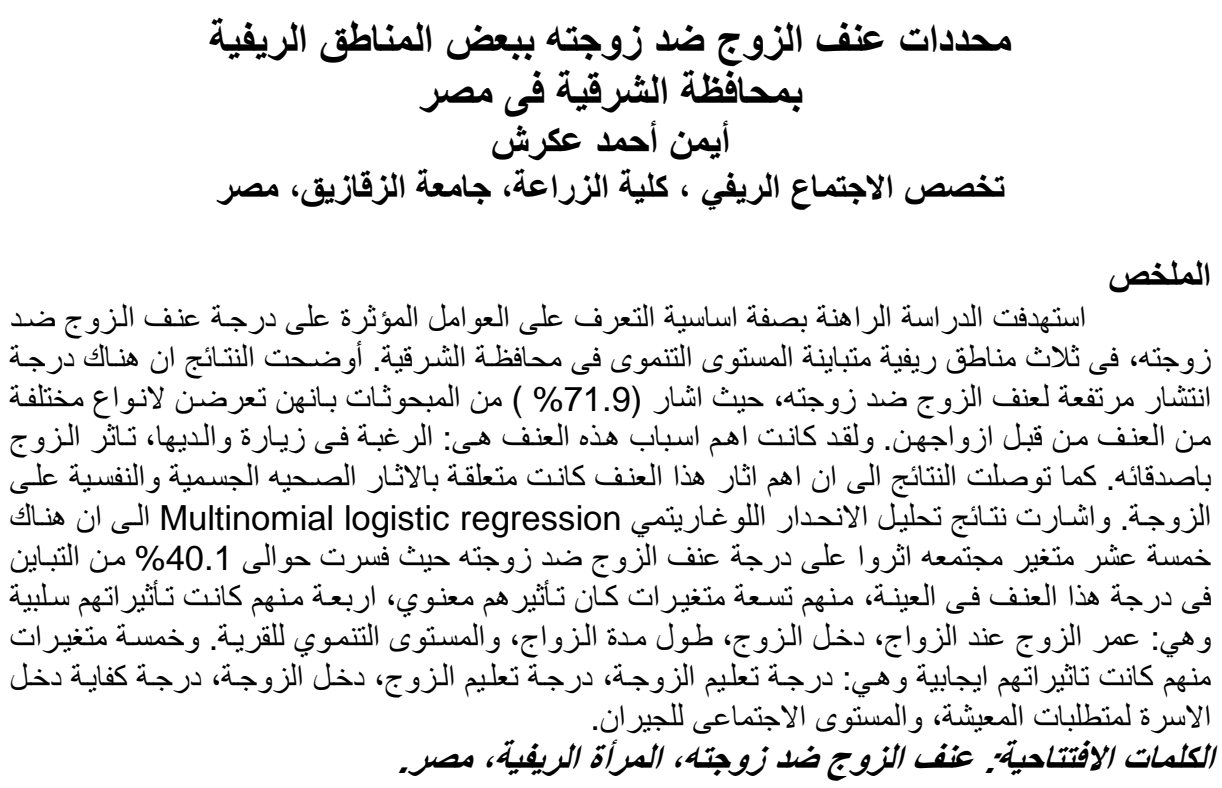